\title{
VLA H53 $\alpha$ radio recombination line observations of the ultraluminous infrared galaxy Arp 220
}

\author{
C. A. Rodríguez-Rico ${ }^{1}$ \\ crodrigu@nrao.edu \\ W. M. Goss ${ }^{2}$ \\ mgoss@nrao. edu \\ F. Viallefond ${ }^{3}$ \\ fviallef @maat . obspm . fr \\ J.-H. Zhao ${ }^{4}$ \\ jzhao@cfa.harvard.edu \\ Y. Gómez ${ }^{1}$ \\ $\mathrm{y} \cdot$ gomez@astrosmo.unam.mx \\ K. R. Anantharamaiah ${ }^{5}$
}

\begin{abstract}
We present high angular resolution $(0,7)$ observations made with the Very Large Array (VLA) of the radio recombination line (RRL) H53 $\alpha$ and radio continuum emission at $43 \mathrm{GHz}$ from the ultraluminous infrared galaxy (ULIRG) Arp 220. The $43 \mathrm{GHz}$ continuum emission shows a compact structure $\left(\sim 2^{\prime \prime}\right)$ with two peaks separated by $\sim 1^{\prime \prime}$, the East $(\mathrm{E})$ and West $(\mathrm{W})$ components, that correspond to each galactic nucleus of the merger. The spectral indices for both
\end{abstract}

\footnotetext{
${ }^{1}$ Centro de Radioastronomía y Astrofísica, UNAM, Campus Morelia, Apdo. Postal 3-72, Morelia, Michoacán 58089, México.

${ }^{2}$ National Radio Astronomy Observatory, Socorro, NM 87801

${ }^{3}$ LERMA, Observatoire de Paris, 61 Av. de l'Observatoire F-75014 Paris

${ }^{4}$ Harvard-Smithsonian Center for Astrophysics, 60 Garden Street, Cambridge, MA 02138

${ }^{5}$ Raman Research Institute, C.V. Raman Avenue, Bangalore, 560 080, India. Deceased 2001, October 29
} 
the $\mathrm{E}$ and $\mathrm{W}$ components, using radio continuum images at 8.3 and $43 \mathrm{GHz}$ are typical of synchrotron emission $(\alpha \sim-1.0)$. Our $43 \mathrm{GHz}$ continuum and $\mathrm{H} 53 \alpha$ line observations confirm the flux densities predicted by the models proposed by Anantharamaiah et al. (2000). This agreement with the models implies the presence of high-density $\left(\sim 10^{5} \mathrm{~cm}^{-3}\right)$ compact HII regions $(\sim 0.1 \mathrm{pc})$ in Arp 220 . The integrated $\mathrm{H} 53 \alpha$ line emission is stronger toward the non-thermal radio continuum peaks, which are also coincident with the peaks of molecular emission of the $\mathrm{H}_{2} \mathrm{CO}$. The coincidence between the integrated $\mathrm{H} 53 \alpha$ and the $\mathrm{H}_{2} \mathrm{CO}$ maser line emission suggests that the recent star forming regions, traced by the high density gas, are located mainly in regions that are close to the two radio continuum peaks. A velocity gradient of $\sim 0.30 \mathrm{~km} \mathrm{~s}^{-1} \mathrm{pc}^{-1}$ in the $\mathrm{H} 53 \alpha \mathrm{RRL}$ is observed toward the E component and a second velocity gradient of $\sim 0.15 \mathrm{~km} \mathrm{~s}^{-1} \mathrm{pc}^{-1}$ is detected toward the $\mathrm{W}$ component. The orientations of these velocity gradients are in agreement with previous $\mathrm{CO}, \mathrm{HI}$ and $\mathrm{OH}$ observations. The kinematics of the high-density ionized gas traced by the $\mathrm{H} 53 \alpha$ line are consistent with two counter rotating disks as suggested by the $\mathrm{CO}$ and $\mathrm{HI}$ observations.

Subject headings: galaxies: general — galaxies: individual(Arp 220) — galaxies: starburst — radio continuum: galaxies — radio lines: galaxies

\section{INTRODUCTION}

Arp 220 (IC 4553/4; UGC 9913; IRAS 15327+2340) has an infrared luminosity of $\sim 1.5 \times 10^{12} \mathrm{~L}_{\odot}$, as determined from IRAS $25-100 \mu \mathrm{m}$ observations (Soifer et al. 1984), and therefore is classified as an ULIRG. At a distance of $\sim 70 \mathrm{Mpc}\left(\mathrm{H}_{o}=75 \mathrm{~km} \mathrm{~s}^{-1} \mathrm{Mpc}^{-1}\right)$, Arp 220 is characterized in the optical by tidal tails interpreted as the signature of a merging process (Surace, Sanders \& Evans 2000). The large visual extinction toward Arp 220, which could be $\mathrm{A}_{V}>100 \mathrm{mag}$ (González-Alfonso et al. 2004), precludes the observations of the nuclear regions at optical and even at infrared frequencies. Radio frequency observations do not suffer from dust extinction, providing a powerful tool to investigate the kinematics, morphology and physical properties of the gas in the dust-obscured regions of Arp 220. Radio observations reveal a double-nucleus system separated by $\sim 1^{\prime \prime}(\sim 350 \mathrm{pc})$ as observed in CO

and HI (Mundell et al. 2001; Sakamoto et al. 1999). The existence of multiple luminous radio supernovae (RSN) in the nuclear regions of Arp 220 suggests that it is mainly starburstpowered (Smith et al. 1998). However, compact OH maser and X-rays emission suggest the presence of AGN activity in the W nucleus (Norris et al. 1985; Lonsdale et al. 1998; Clements et al. 2002). 
High angular resolution (0..5) CO observations carried out by Sakamoto et al. (1999) suggest that the two nuclei are counter-rotating with respect to each other and are embedded in a kiloparsec-size gas disk, which rotates around their dynamical center. The counter-rotation is also observed in higher angular resolution ( 0.22$)$ observations of HI absorption (Mundell et al. 2001), which show that the rotation is not coplanar and proposed a model in which the two nuclei are in the final state of merging. Counter-rotation may provide the mechanism to get rid of angular momentum, a condition for the two nuclei to merge. The senses of the velocity gradients, as determined from $\mathrm{OH}$ maser emission observations (Rovilos et al. 2003), are consistent with CO molecular emission and HI absorption observations (Sakamoto et al. 1999; Mundell et al. 2001).

Arp 220 is the most distant system from which radio recombination lines (RRLs) have been observed (Zhao et al. 1996; Anantharamaiah et al. 2000). VLA observations of the centimeter wavelength RRL H92 $\alpha$ have been reported by Zhao et al. (1996) and Anantharamaiah et al. (2000) with angular resolutions of $4^{\prime \prime}$ and $1^{\prime \prime}$, respectively. Anantharamaiah et al. (2000), using a single dish telescope (IRAM 30m), detected the emission of the millimeter wavelength RRLs H42 $\alpha$, H40 $\alpha$, and H31 $\alpha$ and with the VLA established upper limits for the RRLs H167 $\alpha$ and H165 $\alpha$. Anantharamaiah et al. (2000) constructed a model using three density components of ionized gas in order to reproduce the observed RRL and radio continuum intensities, obtaining a star formation rate (SFR) in Arp 220 of $\sim 240 \mathrm{M}_{\odot} \mathrm{yr}^{-1}$. The existence of a high-density component of ionized gas, as deduced from these models, has been interpreted as evidence for recent star formation in Arp 220. In particular, the RRL H53 $\alpha$ can be used as a tracer of thermal high-density gas $\left(\sim 10^{5} \mathrm{~cm}^{-3}\right)$. The star formation in this system may be supported by multiple starbursts with a high star formation rate.

In this paper we present VLA observations of Arp 220 in the millimeter regime of the RRL H53 $\alpha$ ( $7 \mathrm{~mm})$ as well as the radio continuum at $43 \mathrm{GHz}$ with subarcsecond $\left(00^{\prime \prime} 7\right)$ angular resolution. The observations are described in $\S 2$. In Section 3 the results obtained from the $43 \mathrm{GHz}$ radio continuum and the RRL H53 $\alpha$ are presented. In $\S 4$ the implications of these results are discussed. The conclusions are presented in $\S 5$.

\section{VLA Observations of the $\mathrm{H} 53 \alpha$ line.}

The RRL H53 $\alpha$ (43 GHz) was observed with the VLA in the C configuration on October 13 and 25, 2002. The flux density scales were determined using observations of J1331+305 (1.49 Jy). The fast-switching mode was used in order to track the phase variations induced by the troposphere using cycles of $230 \mathrm{~s}$ on Arp 220 and $40 \mathrm{~s}$ on the phase reference source $\mathrm{J} 1540+147(\sim 1 \mathrm{Jy})$. The bandpass response of the instrument is frequency dependent 
and observations of a bandpass calibrator were required for each frequency window. This bandpass response was corrected using observations of J1229+020 (17.5 $\pm 0.5 \mathrm{Jy}$ ). Five frequency windows, each of these with 15 spectral channels $\left(\sim 22 \mathrm{~km} \mathrm{~s}^{-1}\right.$ each), were used to observe the broad RRL. The total velocity coverage is $\sim 1000 \mathrm{~km} \mathrm{~s}^{-1}$. The different frequency windows were centered at 42114.9, 42135.1, 42164.9, 42185.1 and $42214.9 \mathrm{MHz}$. The on-source integration time was $\sim 2$ hrs for each frequency window. To avoid possible phase decorrelation at $7 \mathrm{~mm}$, the calibration of the data was performed correcting for the phases in a first step and subsequently correcting for both amplitude and phase. The spectral line data were further calibrated by applying the solutions obtained from the self-calibration performed on the continuum channel (which contains the average of the central $75 \%$ of the bandpass) of each frequency window. The spectral line observations of the second day were not combined with those of the first day since the variable content of water vapor in the troposphere caused decorrelation of phases in the second and third frequency windows. A critical step in the reduction of the data is to determine the bandpass shape by normalizing the bandpass using the channel 0 data. Five line images, one for each frequency window of the first day, were produced using a weighting scheme intermediate between natural and uniform to obtain a circular beam of 0.7 . The five line cubes were regridded in frequency and combined into a single line cube in the GIPSY environment. The method used to combine different windows is described by Rodríguez-Rico et al. (2004). The data were then Hanningsmoothed to minimize the Gibbs effect and a final velocity resolution of $\sim 44 \mathrm{~km} \mathrm{~s}^{-1}$ was achieved. The parameters of the observations are given in Table 1. The resulting line cube was further processed using IMLIN from AIPS to subtract the continuum emission. A first order polynomial fit was used for the line-free channels, two on the low-velocity edge and five on the high velocity-edge of the bandpass. Previous Arp 220 observations of the RRL H42 $\alpha$ (Anantharamaiah et al. 2000), CO(2-1) emission line (Sakamoto et al. 1999) and HI absorption (Mundell et al. 2001), show that the full width at zero intensity expected for the $\mathrm{H} 53 \alpha$ line is in the range from $\sim 5000$ to $5700 \mathrm{~km} \mathrm{~s}^{-1}$. The velocity range covered by the $\mathrm{H} 53 \alpha$ line observations is $5100-6100 \mathrm{~km} \mathrm{~s}^{-1}$, consequently loosing the continuum that corresponds to the blueshifted side of the line. We performed a test using the linefree channels on the blueshifted side of the line as a baseline. We have determined that if weak $\mathrm{H} 53 \alpha$ line emission with intensity $<3 \mathrm{mJy}(3 \sigma)$ is present in the velocity range of $5000-5100 \mathrm{~km} \mathrm{~s}^{-1}$ the full width at half maximum (FWHM) is not affected. The fit for the $\mathrm{H} 53 \alpha$ line is in agreement with the fit obtained using the continuum for the low velocity edge of the bandpass. The $\mathrm{H} 53 \alpha(43 \mathrm{GHz})$ data have been compared with the RRL H92 $\alpha$ (8.3 GHz) observations taken by Anantharamaiah et al. (2000) with the VLA in the B array. 


\section{RESULTS}

\subsection{Radio continuum.}

Figure 1 shows the continuum image of Arp 220 at $43 \mathrm{GHz}$ superposed on the continuum image at $8.3 \mathrm{GHz}$, both images with an angular resolution of 0.7 . In this image, the two continuum peaks (E and $\mathrm{W}$ ) are clearly resolved at both frequencies. Using the task IMFIT in AIPS we estimated the properties of these two components. The physical parameters at 8.3 and $43 \mathrm{GHz}$ were obtained integrating over equivalent areas in order to estimate the spectral index $\alpha\left(\mathrm{S}_{\nu} \propto \nu^{\alpha}\right)$ of each component.

Table 2 lists the physical parameters of the continuum components $\mathrm{E}$ and $\mathrm{W}$ determined at $43 \mathrm{GHz}$. The total measured $43 \mathrm{GHz}$ continuum flux density of Arp 220 is $44 \pm 4 \mathrm{mJy}$. The E and $\mathrm{W}$ components have continuum flux densities of $\sim 17$ and $\sim 21$ mJy, respectively. There is also a contribution of $\sim 6 \mathrm{mJy}$ to the total flux density from extended emission that was determined using the task IRING in the AIPS environment by integrating the flux density over an angular scale of $3^{\prime \prime}$ to $8^{\prime \prime}$. This total continuum flux density at $43 \mathrm{GHz}$ is in agreement with the value obtained by interpolating from previous observations at $32 \mathrm{GHz}$ and $97 \mathrm{GHz}$ (Downes \& Solomon 1998, Anantharamaiah et al. 2000). The continuum physical parameters at $8.3 \mathrm{GHz}$ of both the E and W components in Arp 220 are listed in Table 3. The positions of each continuum peak are coincident at 8.3 and $43 \mathrm{GHz}$. The two radio nuclei of Arp 220 are separated by $0 . \prime 9 \pm 0 . \prime 1$ at a position angle of $95^{\circ}$ (E with respect to $\mathrm{N})$. The spectral index between these two frequencies for the $\mathrm{E}$ component is $-0.96 \pm 0.08$ and for the $\mathrm{W}$ component is $-0.97 \pm 0.07$. These spectral indices are typical of non-thermal emission.

\subsection{Radio Recombination Line $\mathbf{H} 53 \alpha$}

We detected H53 $\alpha$ RRL emission (> $3 \mathrm{mJy}$ ) toward both components, E and W, of Arp 220 in the velocity range 5370 to $5845 \mathrm{~km} \mathrm{~s}^{-1}$. The velocity range over which the H53 $\alpha$ line emission is observed agrees with observations of the RRLs H40 $\alpha, \mathrm{H} 42 \alpha$, and H92 $\alpha$ (Anantharamaiah et al. 2000) and the CO(2-1) line (Sakamoto et al. 1999). Figure 2 shows the VLA spectral channel images of $\mathrm{H} 53 \alpha$ line emission observed over the central $\sim 500 \mathrm{pc}$ of Arp 220. Figure 3 shows the velocity-integrated line emission (moment 0) of the H53 line superposed on the moment 0 of the H92 $\alpha$ line. The peaks in the moment 0 of the H53 line emission are nearly coincident $(<0.5)$ with the two radio continuum nuclei. The moment 0 of the H92 $\alpha$ line emission (shown in gray scale) exhibits two peaks that are close to the continuum peaks and are coincident with the H53 $\alpha$ peaks within 0.2 . 
The total integrated spectrum over the region with detectable $\mathrm{H} 53 \alpha$ line emission is shown in Figure 4 along with the $\mathrm{E}$ and $\mathrm{W}$ spectra obtained by integrating over each of these components. A single Gaussian fit was carried out for each line profile. The results from the $\mathrm{H} 53 \alpha$ line fits are listed in Table 4. The peak line flux density in column (2), The FWHM of the line is given in column (3), the heliocentric velocity of the line center in column (4) and the integrated line emission in column (5). Based on the fit parameters for the E and $\mathrm{W}$ components, the peak $\mathrm{H} 53 \alpha$ line flux density for the W component is approximately $30 \%$ stronger than the E component. The $\mathrm{H} 53 \alpha$ line widths and central velocities are similar for the E and W components. The H53 $\alpha$ and H92 $\alpha$ line widths and central velocities determined for the total integrated line emission over Arp 220 are in good agreement with each other. Figure 5 shows the results from radio continuum and RRL emission models taken from Anantharamaiah et al. (2000). The measurements for the total integrated $43 \mathrm{GHz}$ continuum and $\mathrm{H} 53 \alpha$ line emission are shown in Figure $5 \mathrm{a}$ and $5 \mathrm{~b}$, respectively.

Figure 6 shows the H53 $\alpha$ line velocity field (moment 1) for the ionized gas in Arp 220. Based on this $\mathrm{H} 53 \alpha$ velocity field, two velocity gradients are observed toward the $\mathrm{E}$ and $\mathrm{W}$ components. The velocity gradient on the E component is $\sim 0.30 \pm 0.10 \mathrm{~km} \mathrm{~s}^{-1} \mathrm{pc}^{-1}$, oriented along a P.A. of $\sim 30^{\circ}$. The velocity gradient on the $\mathrm{W}$ component has been marginally detected $\left(\sim 0.15 \pm 0.10 \mathrm{~km} \mathrm{~s}^{-1} \mathrm{pc}^{-1}\right)$ along a P.A. of $\sim 260^{\circ}$. Due to the lower velocity resolution of the $\mathrm{H} 92 \alpha$ line $\left(230 \mathrm{~km} \mathrm{~s}^{-1}\right)$, compared to the velocity resolution of the $\mathrm{H} 53 \alpha$ line $\left(44 \mathrm{~km} \mathrm{~s}^{-1}\right.$ ), a detailed comparison of the H53 $\alpha$ and the H92 $\alpha$ velocity fields is not possible.

\section{DISCUSSION}

The measured separation between the two continuum peaks $(0.9 \pm 0.1 \simeq 320 \mathrm{pc})$ and the P.A. of their separation $\left(95^{\circ}\right)$ at $43 \mathrm{GHz}$ are in good agreement with previous measurements $(\sim 350 \mathrm{pc})$ at 5, 15 and $23 \mathrm{GHz}$ (Norris 1988) and $4.8 \mathrm{GHz}$ (Baan \& Haschick 1995). The peak positions at 8.3 and $43 \mathrm{GHz}$ agree at the level of 0 "' 1 . The spectral index for Arp 220, determined using the radio continuum flux density of $\sim 170$ mJy measured at $8.3 \mathrm{GHz}$ and the measurement at $43 \mathrm{GHz}$ of $\sim 44 \mathrm{mJy}$, is $\alpha \sim-1.0$. On the other hand, the spectral index determined using the radio continuum flux density of $\sim 49$ mJy measured at $32 \mathrm{GHz}$ (Baan, Gusten \& Haschick 1986) and the measurement at $43 \mathrm{GHz}$ (this work) of $\sim 44 \mathrm{mJy}$, is $\alpha \simeq 0.36( \pm 0.05)$. Anantharamaiah et al. (2000) estimated that the thermal radio continuum flux density at $5 \mathrm{GHz}$ is $\sim 32 \mathrm{mJy}$. Using a spectral index value $\alpha=-0.1$ for the thermal free-free emission the expected thermal flux density at $43 \mathrm{GHz}$ is $\sim 25 \mathrm{mJy}$, which is $\sim 60 \%$

of the total measured continuum flux density at $43 \mathrm{GHz}$ (see Figure 5). The flattening of the 
spectral index, a value of $\alpha$ closer to zero when the 32 and $43 \mathrm{GHz}$ measurements are used, indicates that the ratio between the thermal emission and non-thermal emission is larger at $43 \mathrm{GHz}$ than at $8.3 \mathrm{GHz}$.

Anantharamaiah et al. (2000) modeled the emission of radio continuum and RRL observations at different frequencies. In these models Anantharamaiah et al. (2000) used three thermally ionized gas components (A1, B1 and D) and non-thermal emission. Components A1 and D are characterized by electron densities of $10^{3} \mathrm{~cm}^{-3}$, while component B1 has a larger electron density of $2.5 \times 10^{5} \mathrm{~cm}^{-3}$. All parameters for each of these components are listed in Table 9 of Anantharamaiah et al. (2000). Figure 5 shows the model results from Anantharamaiah et al. (2000) including the measurements of the H53 $\alpha$ line $\left(\sim 510 \times 10^{-23} \mathrm{~W} \mathrm{~m}^{-2}\right.$ or $\left.3.7 \mathrm{Jy} \mathrm{km} \mathrm{s}^{-1}\right)$ and the continuum flux density at $43 \mathrm{GHz}$ ( $44 \mathrm{mJy})$. The H53 $\alpha$ line and $43 \mathrm{GHz}$ continuum flux densities are in agreement with the values predicted in the Anantharamaiah et al. (2000) models, confirming the presence of a high-density ionized gas component $\left(\sim 10^{5} \mathrm{~cm}^{-3}\right)$ composed of compact HII regions with $\sim 0.1 \mathrm{pc}$ diameter. According to these models the contribution from the dense gas to the H92 $\alpha$ line emission is negligible and the contribution from the lower density gas to the H53 $\alpha$ line is $<10 \%$.

On the other hand, the models of Anantharamaiah et al. (2000) indicate that $\sim 50 \%$ of the $\mathrm{H} 53 \alpha$ line emission in the high-density ionized gas is due to internal stimulated line emission. The spatial distribution of the $\mathrm{H} 53 \alpha$ and the H92 $\alpha$ lines is similar toward the E component as can be seen in Figure 3. However, a remarkable difference is observed toward the $\mathrm{W}$ component where the H92 $\alpha$ line emission is more extended than the H53 $\alpha$ line emission. Near the continuum peaks, the spatial distribution of the H53 $\alpha$ and H92 $\alpha$ RRLs emission suggests that the recent star formation is taking place mostly in two regions. These two regions of line emission are slightly displaced from the respective continuum peaks: the $\mathrm{W}$ line peak is $0.2 \pm 00^{\prime \prime} 1$ to the $\mathrm{S}$ of its associated continuum peak and the E line peak is $0.3 \pm 00^{\prime \prime} 1$ to the $\mathrm{N}$ of its associated continuum peak. According to the integrated H53 $\alpha$ line emission (see Figure 3), the bulk of high-density ionized gas is concentrated in these two regions. This result is supported by the spatial correlation between the two velocityintegrated $\mathrm{H} 53 \alpha$ line emission peaks and the peaks of the formaldehyde $\left(\mathrm{H}_{2} \mathrm{CO}\right)$ molecular emission (Baan \& Haschick 1995).

The models of Anantharamaiah et al. (2000) derived a SFR in Arp 220 of $240 \mathrm{M}_{\odot} \mathrm{yr}^{-1}$, assuming a mass range of $1-100 \mathrm{M}_{\odot}$ in the Miller-Scalo initial mass function (IMF). According to these models, the SFR could be as low as $90 \mathrm{M}_{\odot} \mathrm{yr}^{-1}$ if the upper mass limit in the IMF is reduced to $60 \mathrm{M}_{\odot}$ and the Salpeter IMF is used. Given that the radio continuum and the velocity-integrated line flux density are in agreement with the values 
expected from the models of Anantharamaiah et al. (2000), the value for the SFR could be in the range of 90 to $240 \mathrm{M}_{\odot} \mathrm{yr}^{-1}$ and the mass of high-density ionized gas $\left(\sim 10^{5} \mathrm{~cm}^{-3}\right)$ is between $10^{3}$ and $10^{4} \mathrm{M}_{\odot}$.

The orientations of the $\mathrm{H} 53 \alpha$ velocity gradients in Arp 220 , with P.A. $\sim 30^{\circ}$ in the E component and P.A. $\sim 260^{\circ}$ in the $\mathrm{W}$ component agree with the $\mathrm{CO}$ and $\mathrm{HI}$ observations (Sakamoto et al. 1999; Mundell et al. 2001). Based on the P.A. of these two velocity gradients, the CO(2-1) line observations were interpreted by Sakamoto et al. (1999) as evidence of two counter-rotating disks in Arp 220. An alternative model that consists of a warped gas disk that resulted from a merger of two spiral galaxies was also proposed by Eckart \& Downes (2001). The angular resolution achieved in the H53 $\alpha$ line observations $\left(0{ }^{\prime \prime} 7\right)$ is insufficient to discern between these two models. The velocity structure of the ionized gas based on the $\mathrm{H} 53 \alpha$ data show two velocity gradients in the two components of Arp 220. On the E component the $\mathrm{H} 53 \alpha$ velocity gradient is $\sim 0.30 \mathrm{~km} \mathrm{~s}^{-1} \mathrm{pc}^{-1}$. The HI velocity gradient on the E component is $1.01 \pm 0.02 \mathrm{~km} \mathrm{~s}^{-1} \mathrm{pc}^{-1}$ (Mundell et al. 2001) and the $\mathrm{OH}$ velocity gradient is $0.32 \pm 0.03 \mathrm{~km} \mathrm{~s}^{-1} \mathrm{pc}^{-1}$ (Rovilos et al. 2003).

The velocity gradient on the E component implies a virial mass of $\sim 8 \times 10^{7}\left(\sin ^{-2} i\right) \mathrm{M}_{\odot}$ in a disk of radius $\sim 180 \mathrm{pc}$ ( $i$ is the inclination). The total mass calculated from $\mathrm{OH}$ observations, for a disk of radius $\sim 80 \mathrm{pc}$, is $\sim 10^{7} \mathrm{M}_{\odot}$ (Rovilos et al. 2003). The value derived from the $\mathrm{OH}$ observations is consitent with the $\mathrm{H} 53 \alpha$ line observations when the different

sizes are taken into account. Based on HI and CO (Sakamoto et al. 1999; Mundell et al. 2001) observations, the estimates of the total mass are $\sim 10^{9} \mathrm{M}_{\odot}$. The total mass estimated using $\mathrm{CO}$ and $\mathrm{HI}$ observations is two orders of magnitude larger than the total mass calculated from these $\mathrm{H} 53 \alpha$ line observations. The smaller estimates obtained from the H53 $\alpha$ line and $\mathrm{OH}$ observations are explained if the molecular $\mathrm{CO}$ and neutral $\mathrm{HI}$ gas are distributed over a larger region compared to the ionized gas. In the direction of the $\mathrm{W}$ component, the $\mathrm{H} 53 \alpha$ velocity gradient is a factor of 10 less than observed in $\mathrm{HI}$ absorption and $\mathrm{OH}$ maser emission (Mundell et al. 2001; Rovilos et al. 2003). However, the orientation and sense of the velocity gradient in $\mathrm{H} 53 \alpha$ are consistent with those determined from the $\mathrm{CO}, \mathrm{HI}$ and $\mathrm{OH}$ observations (Sakamoto et al. 1999; Mundell et al. 2001; Rovilos et al. 2003).

\section{CONCLUSIONS.}

We have observed the RRL $\mathrm{H} 53 \alpha$ and radio continuum at $43 \mathrm{GHz}$ toward the ULIRG Arp 220 with high angular resolution (0.'7) using the VLA. The $43 \mathrm{GHz}$ radio continuum and the RRL H53 $\alpha$ have been compared with observations of the $8.3 \mathrm{GHz}$ radio continuum and H92 $\alpha$ line (Anantharamaiah et al. 2000). 
- The total $43 \mathrm{GHz}$ radio continuum flux density of Arp 220 is $44 \pm 4 \mathrm{mJy}$. The morphological characteristics observed in the radio continuum at $43 \mathrm{GHz}$ agree with previous radio observations made in the range from 1.4 to $23 \mathrm{GHz}$. In the radio continuum at $43 \mathrm{GHz}$, Arp 220 exhibits a double-nucleus system that has been clearly resolved with a separation of the two radio continuum peaks of $\sim 1^{\prime \prime}$ at P.A. of $95^{\circ}$.

- We have determined the spectral indices for both the $\mathrm{E}$ and $\mathrm{W}$ components, using radio continuum flux densities at 8.3 and $43 \mathrm{GHz}$. Both components have spectral indices typical of synchrotron emission $(\alpha \sim-0.9)$, as expected from extrapolation from the centimeter wavelength range.

- Based on $43 \mathrm{GHz}$ VLA data, we confirm the predictions of the models made by Anantharamaiah et al. (2000) for RRLs. The integrated H53 $\alpha$ line flux density is about a factor of $\sim 50$ times larger than the integrated H92 $\alpha$ line flux density, in agreement with the predictions of Anantharamaiah et al. (2000). Thus, the H53 $\alpha$ line traces the high-density $\left(\sim 10^{5} \mathrm{~cm}^{-3}\right)$ compact HII regions $(\sim 0.1 \mathrm{pc})$ in Arp 220 .

- The spatial distributions of the $\mathrm{H} 53 \alpha$ and the $\mathrm{H} 92 \alpha$ line emission are similar in the direction of the $\mathrm{E}$ component. On the $\mathrm{W}$ component the low density $\left(\sim 10^{3} \mathrm{~cm}^{-3}\right)$ ionized gas component is more extended than the high density $\left(\sim 10^{5} \mathrm{~cm}^{-3}\right)$ component.

- The kinematic and spatial distribution behavior as observed in the RRL H53 $\alpha$ is in agreement with results reported from CO and HI observations (Sakamoto et al. 1999; Mundell et al. 2001), supporting the counter-rotation of two disks in Arp 220 at smaller scales.

The original version was published in the Astrophysical Journal, volume 633, page 198 in year 2005. This new version is submitted with the corrected velocity scale. In Figures 2, 4 and 6 the values for radial velocities were incorrect. All velocities were increased by $245 \mathrm{~km} \mathrm{~s}^{-1}$ to correct values to heliocentric values (optical definition). To obtain the velocities using radio definition, add $145 \mathrm{~km} \mathrm{~s}^{-1}$. Thus the central velocities in Table 4 should be $5495 \mathrm{~km} \mathrm{~s}^{-1}$ (optical definition) or $5395 \mathrm{~km} \mathrm{~s}^{-1}$ (radio definition). This velocity shift was introduced in the frequency reprojection process. The velocity definition in Table 4 is the optical heliocentric definition, as is used by K. R. Anantharamaiah et al. (ApJ, 537, 613 [2000]). All other quantities in the paper remain unchanged, e.g. the radio recombination line and radio continuum integrated properties, as well as distributions.

The National Radio Astronomy Observatory is a facility of the National Science Foundation operated under cooperative agreement by Associated Universities, Inc. CR and YG 
acknowledge the support from UNAM and CONACyT, México. The authors thank the referee for helpful comments.

\section{REFERENCES}

Anantharamaiah, K. R., Viallefond, F., Mohan, N. R., Goss, W. M., \& Zhao, J. H., 2000, ApJ, 537, 613

Baan, W. A., 1985, Nature, 315, 26

Baan, W. A., Gusten, R. \& Haschick, A. D., 1986, ApJ, 305, 830

Baan, W. A., \& Haschick, A. D., 1995, ApJ, 454, 745

Baan, W. A., \& Haschick, A. D., 1984, ApJ, 279, 541

Carilli, C. L., Holdaway, M. A., Ho, P. T. P., De Pree, C. G., 1992, ApJ, 399, L59

Clements, D. L., \& Baker, A. C., 1996, A\&A, 314, L5

Downes, D., \& Solomon, P. M., 1998, ApJ, 507, 615

Eckart, A., \& Downes, D., 2001, ApJ, 551, 730

Genzel, R., et al. 1998, ApJ, 498, 579

Gonzalez-Alfonso, E., Smith, H. A., Fischer, J., \& Cernicharo, J., 2004, ApJ, 613, 247

Kennicutt, 1998, ARAA

Lonsdale, C. J., Lonsdale, C. J., Diamond, P. J., \& Smith, H. E., 1998, ApJ, 493, 13

Mundell, C. G., Ferruit, P., \& Pedlar, A., 2001, ApJ, 560, 168

Norris, R. P., Baan, W. A., Haschick, A. D., Diamond, P. J., \& Booth, R. S., 1985, MNRAS, 213, 821

Norris, R. P., 1988, MNRAS, 230, 345

Rodríguez-Rico, C. A., Viallefond, F., Zhao, J.-H., Goss, W. M., \& Anantharamaiah, K. R., 2004, ApJ, 616, 783

Rovilos, E., Diamond, P. J., Lonsdale, C. J., Lonsdale, C. J., Smith, H. E., 2003, MNRAS, 342,373 
Sakamoto, K., Scoville, N. Z., Yun, M. S., Crosas, M., Genzel, R., \& Tacconi, L. J., 1999, ApJ, 514, 68

Sanders, D. B., \& Mirabel, I. F., 1996, ARA\&A, 34, 749

Scoville, N. Z., Sargent, A. I., Sanders, D. B., \& Soifer, B. T., 1991, ApJL, 366, 5

Scoville, N. Z. et al., 1998, ApJ, 492, 107

Smith, H. E., Lonsdale, C. J., Lonsdale, C. J., \& Diamond, P. J., 1998, 493, 17

Soifer, B. T., et al. 1984, ApJ, 283, 1

Solomon, P. M., Downes, D., \& Radford, S. J. E., 1992, ApJL, 387, 55

Zhao, J. H., Anantharamaiah, K. R., Goss, W. M., \& Viallefond, F., 1996, ApJ, 472, 54

This preprint was prepared with the AAS LATEX macros v5.2. 
Table 1. ObServational Parameters For Arp 220

\begin{tabular}{|c|c|}
\hline Parameter & H53 $\alpha$ Line \\
\hline Right ascension (J2000) . & 153457.28 \\
\hline Declination $(\mathrm{J} 2000) \ldots$... & 233011.9 \\
\hline Angular resolution............ & 0.7 \\
\hline Total observing duration (hr). & 13 \\
\hline Bandwidth $(\mathrm{MHz}) \ldots . . . . . .$. & 150 \\
\hline Number of spectral channels..... & 45 \\
\hline Center $\mathrm{V}_{\mathrm{Hel}}\left(\mathrm{km} \mathrm{s}^{-1}\right) \ldots \ldots \ldots$ & 5500 \\
\hline Velocity coverage $\left(\mathrm{km} \mathrm{s}^{-1}\right) \ldots$ & 1000 \\
\hline Velocity resolution $\left(\mathrm{km} \mathrm{s}^{-1}\right)$. & 44 \\
\hline Amplitude calibrator $\ldots \ldots \ldots \ldots \ldots \ldots$ & $\mathrm{J} 1331+305$ \\
\hline Phase calibrator $\ldots \ldots \ldots \ldots \ldots \ldots \ldots$ & $\mathrm{J} 1540+147$ \\
\hline Bandpass calibrator $\ldots \ldots \ldots \ldots \ldots \ldots$ & $\mathrm{J} 1229+020$ \\
\hline RMS line noise per channel (mJy/beam) & 1 \\
\hline RMS, continuum (mJy/beam) .......... & 0.3 \\
\hline
\end{tabular}


Table 2. RESULTS FROM GAUSSIAN FITTING TO THE CONTINUUM EMISSION AT 43 GHZ OF ARP 220.

\begin{tabular}{ccccc}
\hline \hline Feature & RA $(\mathrm{J} 2000)$ & $\operatorname{DEC}(\mathrm{J} 2000)$ & Size $^{\mathrm{a}}$, P.A. & $\mathrm{S}_{C}(\mathrm{mJy})$ \\
\hline Arp 220 E & $15^{h} 34^{m} 57.28 \pm 0.01$ & $23^{\circ} 30^{\prime} 11^{\prime \prime} 3 \pm 0^{\prime \prime} 1$ & $0^{\prime \prime} 5 \times 0 \times 0^{\prime \prime} 3,89^{\circ}$ & $17 \pm 2$ \\
Arp 220 W & $15^{h} 34^{m} 57^{s} .22 \pm 0.01$ & $23^{\circ} 30^{\prime} 11^{\prime \prime} 4 \pm 00^{\prime \prime} 1$ & $0^{\prime \prime} 4 \times 0^{\prime \prime} 2,114^{\circ}$ & $21 \pm 2$ \\
\hline
\end{tabular}

${ }^{a}$ Deconvolved angular size. 
Table 3. RESUlts FROM GAUSSIAN FITTING TO THE CONTINUUM EMISSION AT 8.3 GHZ OF ARP $220^{\mathrm{a}}$.

\begin{tabular}{ccccc}
\hline \hline Feature & RA $(\mathrm{J} 2000)$ & DEC $(J 2000)$ & Size $^{\mathrm{b}}$, P.A. & S $_{C}(\mathrm{mJy})^{\mathrm{c}}$ \\
\hline Arp 220 E & $15^{h} 34^{m} 57^{s} .29 \pm 0.01$ & $23^{\circ} 30^{\prime} 11^{\prime \prime} 3 \pm 0.1$ & $0^{\prime \prime} 5 \times 0 \times 0^{\prime \prime} 4,88^{\circ}$ & $77 \pm 1$ \\
Arp 220 W & $15^{h} 34^{m} 57^{\prime} .22 \pm 0.01$ & $23^{\circ} 30^{\prime} 11^{\prime \prime} .5 \pm 0.1$ & $0^{\prime \prime} 5 \times 0^{\prime \prime} .3,107^{\circ}$ & $89 \pm 1$ \\
\hline
\end{tabular}

a Observations at $8.3 \mathrm{GHz}$ made by Anantharamaiah et al. (2000).

${ }^{\mathrm{b}}$ Deconvolved angular size.

${ }^{\mathrm{c}}$ Continuum flux densities were measured in the same area as the $43 \mathrm{GHz}$ continuum flux densities listed in Table 2. 
Table 4. RESULTS FROM GAUSSIAN FITTING TO THE H53 $\alpha$ LINE EMISSION OF ARP 220.

\begin{tabular}{ccccc}
\hline \hline Feature & $\begin{array}{c}\mathrm{S}_{P} \\
(\mathrm{mJy})\end{array}$ & $\begin{array}{c}\Delta \mathrm{V}_{F W H M} \\
\left(\mathrm{~km} \mathrm{~s}^{-1}\right)\end{array}$ & $\begin{array}{c}\mathrm{V}_{\text {Helio }} \\
\left(\mathrm{km} \mathrm{s}^{-1}\right)\end{array}$ & $\begin{array}{c}1.07\left(\mathrm{~S}_{P} \Delta \mathrm{V}_{F W H M}\right) \\
\left(\mathrm{W} \mathrm{m}^{-2} \times 10^{-23}\right)\end{array}$ \\
\hline Arp 220 E $\ldots$ & $6 \pm 1$ & $235 \pm 20$ & $5485 \pm 10$ & $220 \pm 40$ \\
Arp 220 W $\ldots$ & $8 \pm 1$ & $265 \pm 30$ & $5515 \pm 10$ & $320 \pm 50$ \\
Arp 220 E+W & $16 \pm 2$ & $230 \pm 20$ & $5495 \pm 10$ & $560 \pm 80$ \\
\hline
\end{tabular}




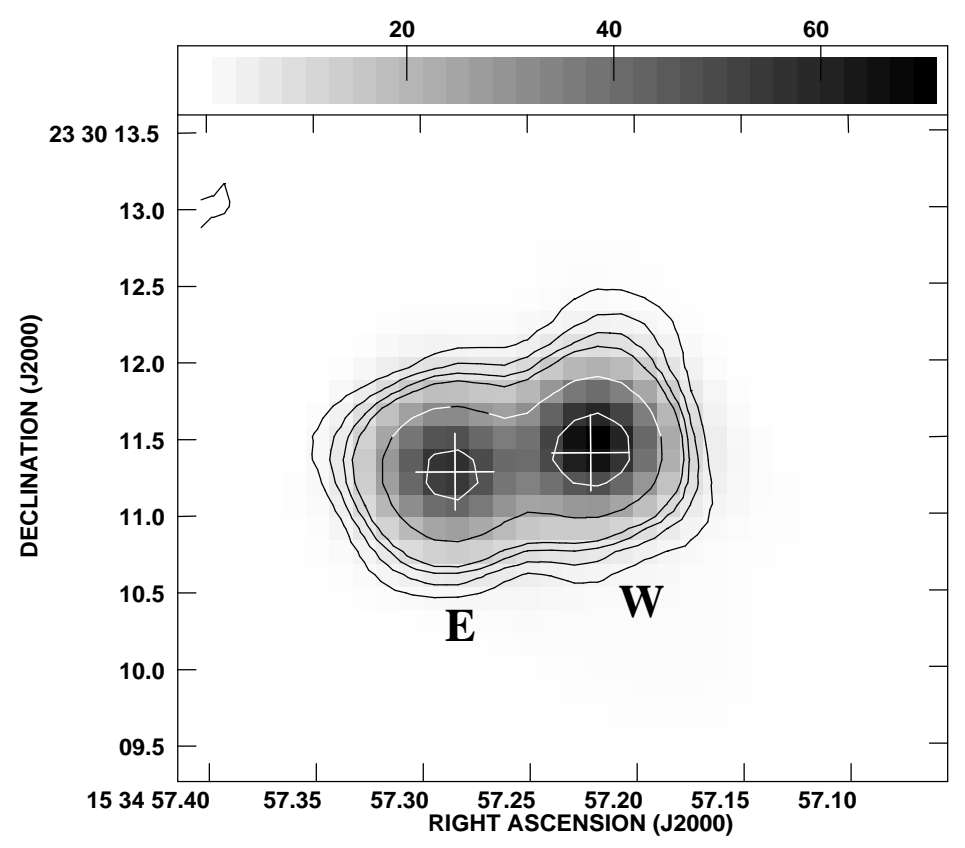

Fig. 1.- Radio continuum image of Arp 220 at $43 \mathrm{GHz}$ (contours) made using VLA observations in the $\mathrm{C}$ array superposed on the continuum image at $8.3 \mathrm{GHz}$ (gray scale) using the VLA in the B array, both images made with angular resolution of 0.7 . The contour levels $(43 \mathrm{GHz})$ are drawn at $-3,3,5,7,9,18$, and 36 times the rms noise $0.3 \mathrm{mJy} \mathrm{beam}^{-1}$. The gray scale $(8.3 \mathrm{GHz})$ ranges from 0.15 to $70 \mathrm{mJy}^{\text {beam }}{ }^{-1}$. The crosses mark the peak positions (given in Table 2) of the $43 \mathrm{GHz}$ continuum. The size of the crosses are 0.5 , which is about five times the error position of the radio continuum peaks. 


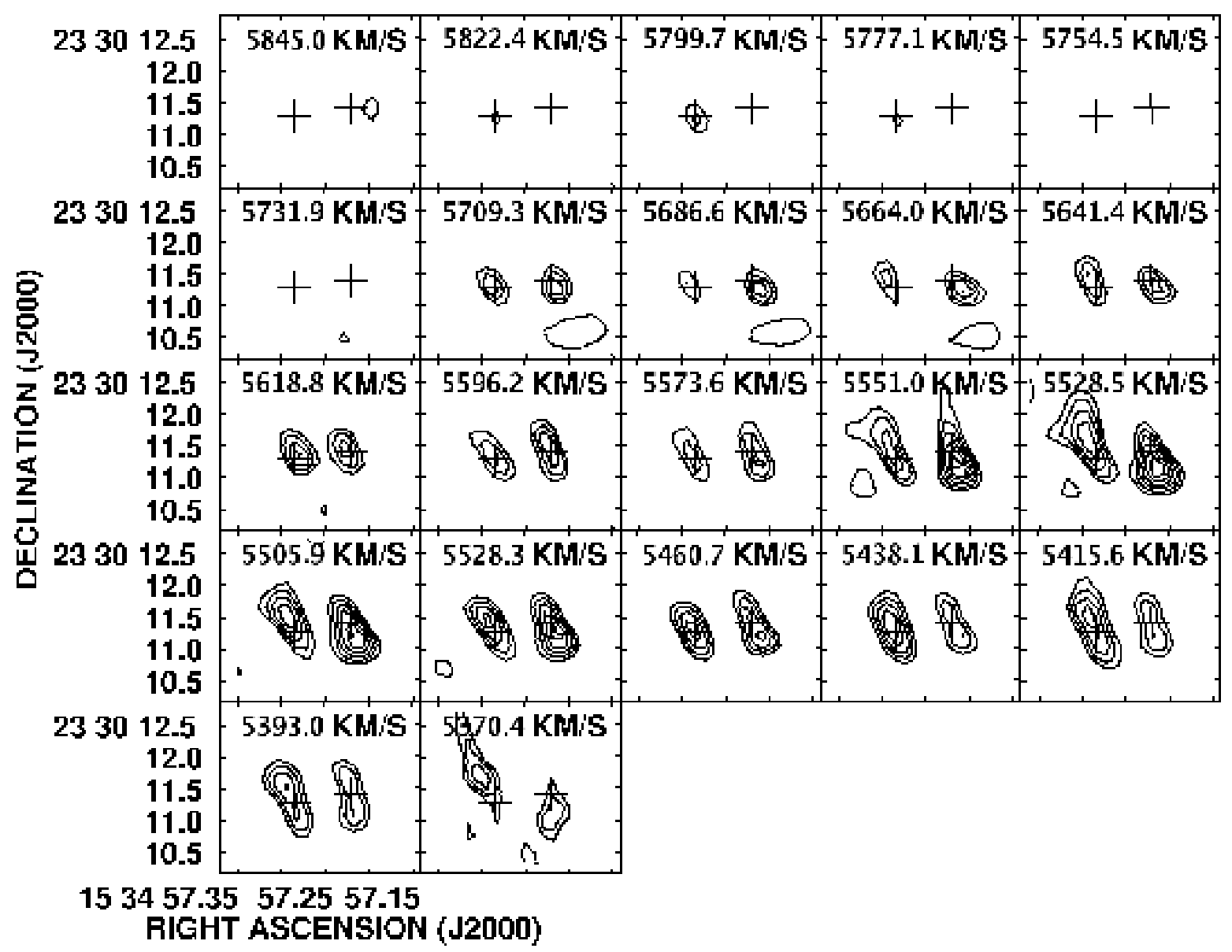

Fig. 2.- H53 $\alpha$ continuum subtracted channel images from Arp 220 labeled according to the central heliocentric velocity of each channel. Contours are -3, 3, 4, 5, 6, 7, 8, 9, 10 and 11 times 1 mJy beam ${ }^{-1}$, the rms noise. The crosses show the position of the two $43 \mathrm{GHz}$ continuum peaks. The synthesized beam is 0.7 FWHM. 


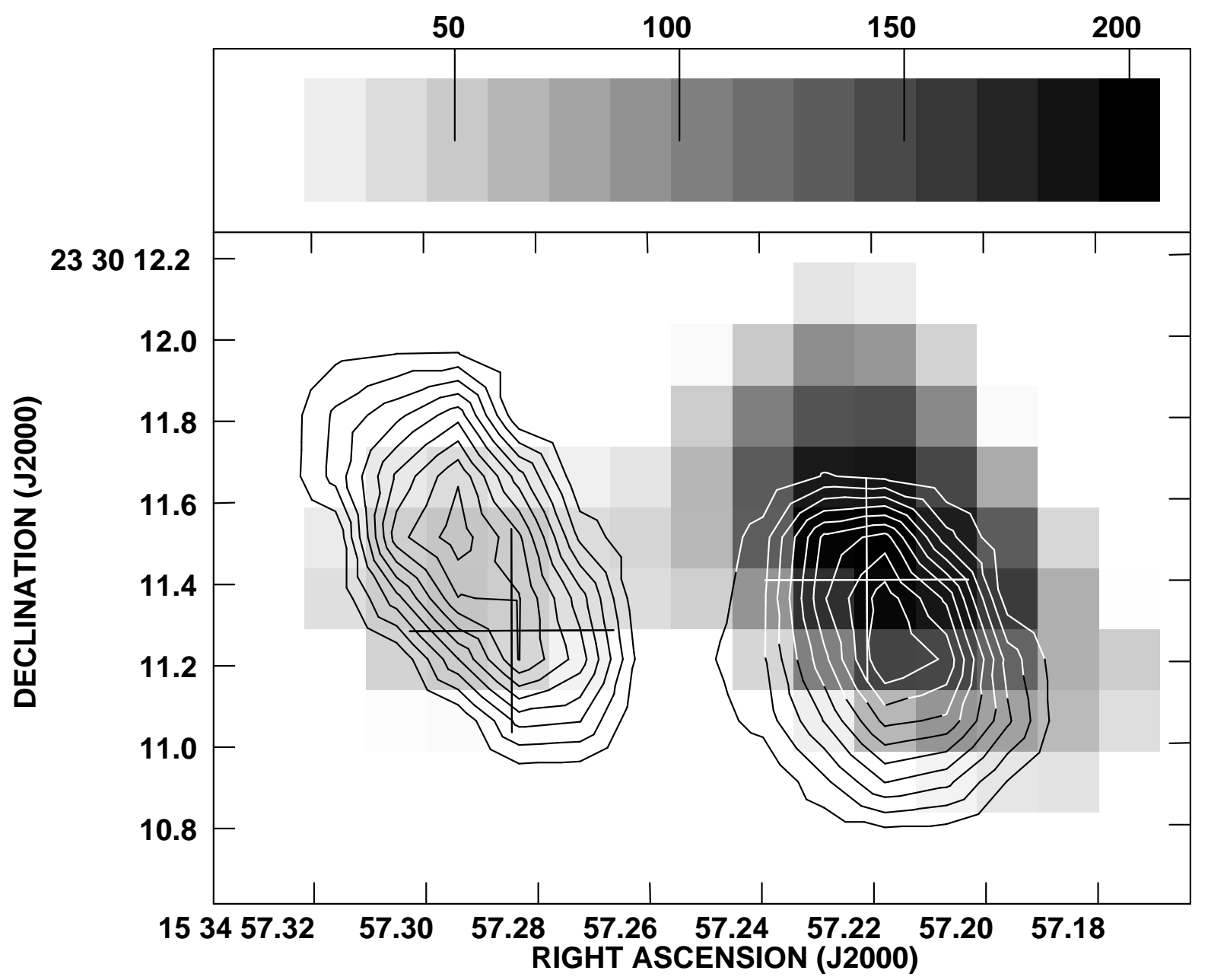

Fig. 3.- Integrated H53 $\alpha$ line emission (moment 0) from Arp 220 in contours with integrated H92 $\alpha$ line emission (moment 0) superposed in gray scale. Contour levels are 10, 30, 50, 70 , and $90 \%$ of the peak $\left(1.3 \mathrm{Jy}_{\mathrm{beam}}^{-1} \mathrm{~km} \mathrm{~s}^{-1}\right)$. The gray scale covers the range $0-$ $200 \mathrm{Jy} \mathrm{beam}^{-1} \mathrm{~km} \mathrm{~s}^{-1}$. The crosses show the position of the two $43 \mathrm{GHz}$ continuum peaks. The synthesized beam for both RRLs is 0.'7 FWHM. 

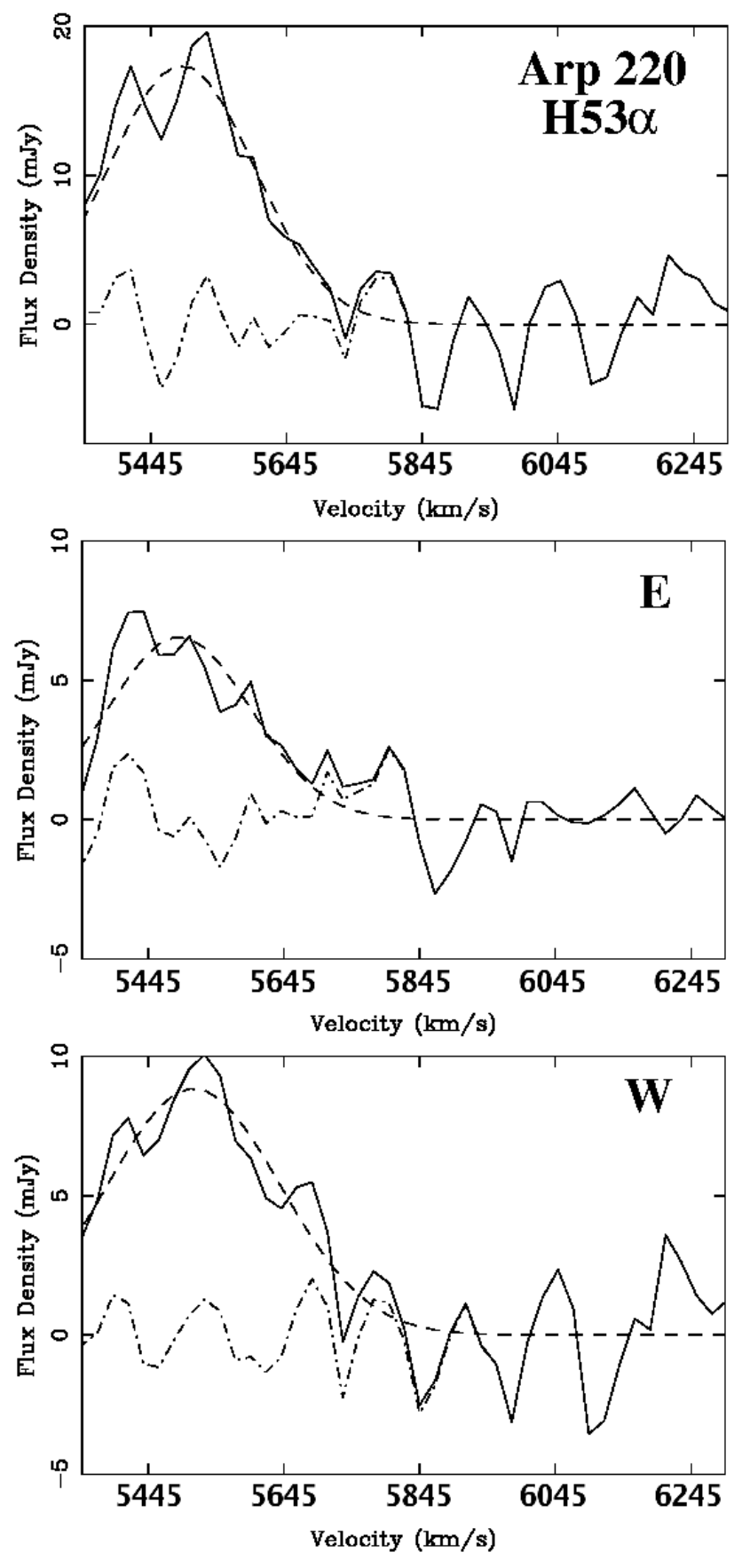

Fig. 4.- H53 $\alpha$ recombination line profiles in Arp 220 observed using the VLA integrated over the region shown in contours in Figure 1 (top), over the E component (middle) and over the $\mathrm{W}$ component (bottom). The dashed line shows the resulting Gaussian fit to the data and the dashed-dotted line indicates the residuals from the Gaussian fit. 


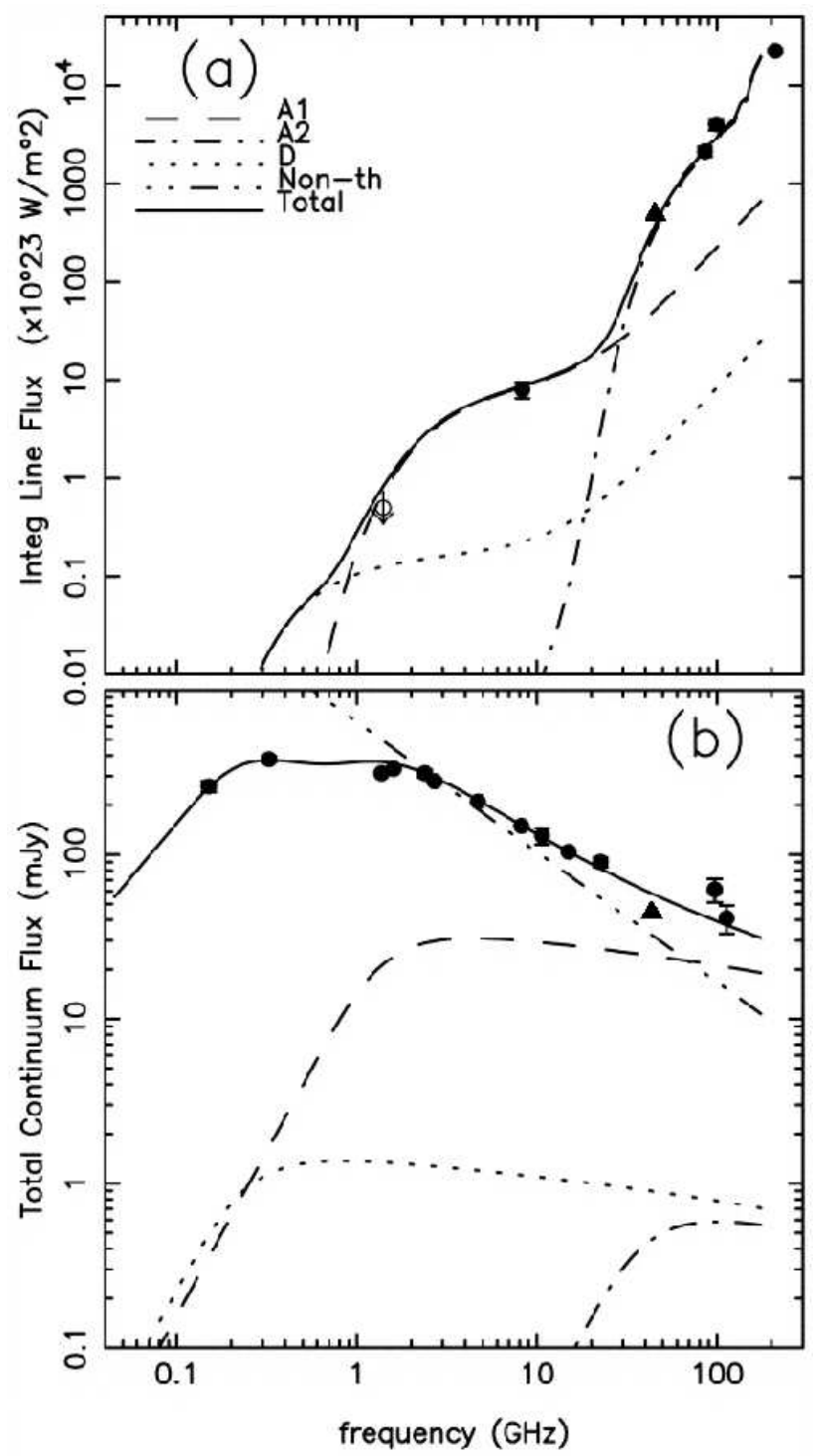

Fig. 5.- Continuum and RRL model results for three ionized gas components (A1, A2, and D) in Arp 220 by Anantharamaiah et al. (2000). The recombination line and continuum flux densities are shown in (a) and (b), respectively. The contribution from the different components are shown and the parameters for each component are given in Table 9 of Anantharamaiah et al. (2000). The observed line data points are listed in Table 2 and 4 of Anantharamaiah et al. (2000). The filled triangles indicate the velocity integrated H53 $\alpha$ line flux density in (a) and the $43 \mathrm{GHz}$ continuum flux density in (b) obtained in the current observations. The size of the triangles represent the $1 \sigma$ error for the measured values. 


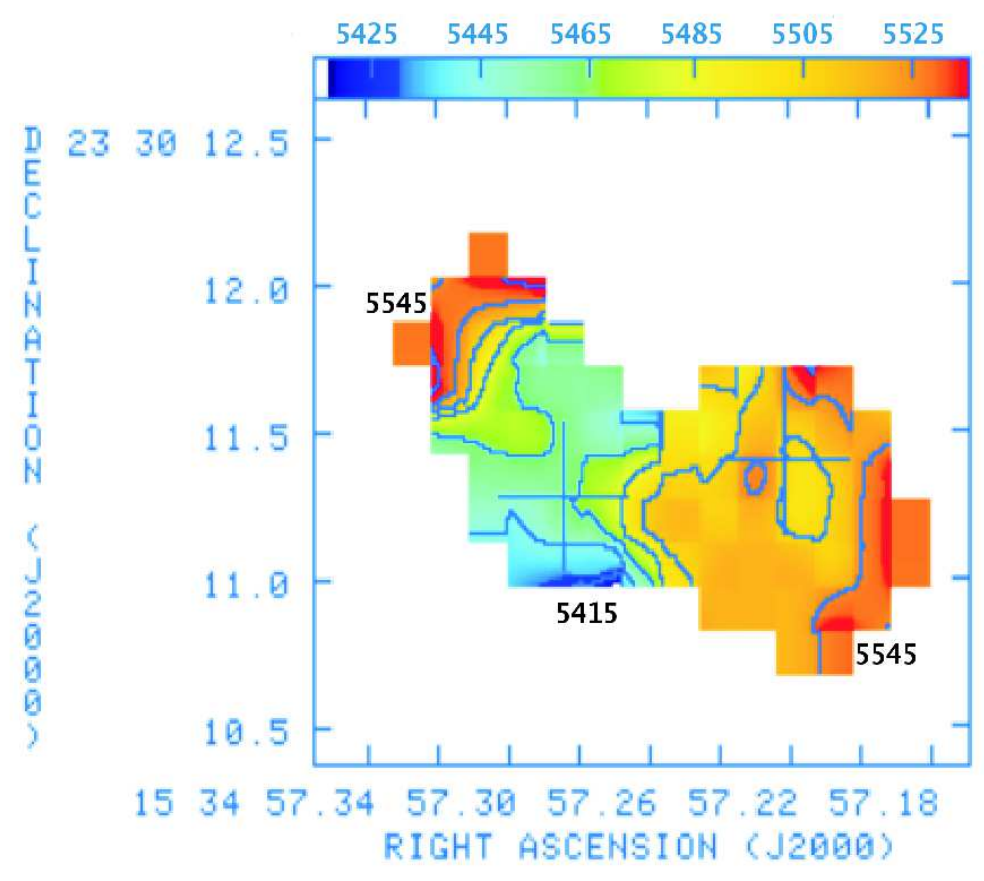

Fig. 6.- Velocity field of Arp 220 as observed in the RRL H53 $\alpha$ with the VLA. The color scale shows the velocity field in the range from 5415 to $5615 \mathrm{~km} \mathrm{~s}^{-1}$. Contour levels are $5425,5445, \ldots, 5605 \mathrm{~km} \mathrm{~s}^{-1}$ in steps of $20 \mathrm{~km} \mathrm{~s}^{-1}$. The crosses show the position of the two $43 \mathrm{GHz}$ continuum peaks. The synthesized beam is 0."7 FWHM. 